\title{
Effective killing of Borrelia burgdorferi in vitro with novel herbal compounds
}

\author{
Kati Karvonen* and Leona Gilbert \\ Department of Biological and Environmental Science and Nanoscience Center, PO Box 35, FI-40014, University of Jyvaskyla, Finland
}

\begin{abstract}
Introduction: The tick-borne disease Lyme Borreliosis is caused by Borrelia bacteria. The disease can persist even after treatment with antibiotics, which is why other methods of treatment are needed. Herbal compounds and phytochemicals have been recently examined in relation to eradicating Borrelia bacteria in vitro.

Objective: The possible antimicrobial effect of two novel compounds, Biocidin ${ }^{\circledR}$ Liquid and LSF Broad-Spectrum Liposomal formulas, was examined in the hopes of discovering an alternative method for eradication of Borrelia bacteria.

Methods: The minimum inhibitory concentrations (MICs) and minimum bacterial deaths (MBDs), as well as, time-kill effect of each compound were utilized in the study.

Results: The Liquid formula effectively killed the spirochetes with 1:10 dilution, while the MIC for the Liposomal formula was 1:25. Moreover, the MIC for both compounds with Round Bodies was 1:50 and for biofilms 1:10. Though long-term effect (MBD) was seen only with 1:5 dilutions for both formulas. Additionally, the killing effect of each compound was observed already at 10 min post-treatment.
\end{abstract}

Conclusion: The study conducted here provides new insight into the antimicrobial effect of herbal compounds. Furthermore, studies such as these are required in order to discover possible alternatives to antibiotics in the battle against Borrelia infections.

Abbreviations: B. burgdorferi: Borrelia burgdorferi; MIC: minimum inhibitory concentration; MBD: minimum bacterial death; RB: Round Body.

\section{Introduction}

The spirochete bacteria Borrelia burgdorferi, found in Ixodes ticks, is the causative agent for Lyme Borreliosis [1]. Unfavorable conditions, such as changes in $\mathrm{pH}$, nutritional depletion, even antibiotics can lead the pleomorphic bacteria to reversibly alter their form into Round Bodies (RBs) or biofilms [2,3]. Furthermore, the immune system reacts differently to the pleomorphic forms with macrophages digesting and processing RBs differently than spirochetes [4]. Hence, examinations of B. burgdorferi need to include these various forms.

Borrelia infected patients report signs and symptoms ranging from skin inflammation, arthritis and neurological or cardiac impairments [5]. Treatment with antibiotics early on the infection can clear the pathogen from the body, however, post-treatment persistence by the bacteria as asymptomatic or with a multiplicity of symptoms can occur [5]. It has been suggested that the different pleomorphic forms of Borrelia are involved in the avoidance of the immune system, and thus, in the persistence of the disease [6].

Current treatment for Lyme borreliosis relies solely on antibiotics [5]. However, studies on antibiotic efficiency with different pleomorphic forms of Borrelia have shown to demonstrate varying effectiveness of antibiotics in killing the bacteria [6,7]. Therefore, new approaches for better treatment are required.

For centuries herbal compounds have been used as remedies for various ailments. Recently, the antimicrobial possibilities of a variety of phytochemicals and herbal extracts against Borrelia have been studied [8-10]. Hence, studies on natural composites might offer new possibilities for remedies to Lyme Borreliosis. Here, two commercially available herbal compounds, Biocidin ${ }^{\circledR}$ Liquid and LSF Broad-Spectrum Liposomal formulas, were tested for their efficiency in eliminating different pleomorphic forms of B. burgdorferi in vitro.

\section{Materials and methods}

\section{Bacterial strain, culturing conditions and test compounds}

All experiments were conducted with infectious, fluorescent $B$. burgdorferi strain GCB726 with GFP, which was graciously provided by Georges Chaconas, University of Calgary, Canada [11]. BarbourStoenner-Kelly medium (BSK II) [12], without gelatin and supplemented with $6 \%$ heat inactivated rabbit serum (Sigma-Aldrich, St. Louis, USA) was used in the culturing of cells at $+37^{\circ} \mathrm{C}$. Low-passage number cells ( $\leq$ passage 8 ) were used in all of the experiments.

The tested compounds, Biocidin Liquid formula and Biocidin LSF Broad-Spectrum Liposomal formula, were acquired from Bio-Botanical Research Inc. (CA, USA). As negative controls for growth $100 \mu \mathrm{g} / \mathrm{ml}$ of doxycycline (Hexal', Germany) and $0.02 \% \mathrm{H}_{2} \mathrm{O}_{2}$ (Sigma-Aldrich) were used. Untreated cells were a positive control for growth.

${ }^{*}$ Correspondence to: Kati Karvonen, M.Sc, Department of Biological and Environmental Science and Nanoscience Center, PO Box 35, FI-40014, University of Jyvaskyla, Finland, E-mail: katis.karvonen@jyu.fi

Key words: Borrelia burgdorferi, pleomorphic form, antimicrobial, persister

Received: December 18, 2018; Accepted: December 28, 2018; Published: December 31, 2018 


\section{Minimum inhibitory concentration and minimum bacterial death experiments}

The minimum inhibitory concentrations (MICs) of Biocidin Liquid and Liposomal formulas were determined by incubating $15 \mathrm{x}$ $10^{6} \mathrm{~B}$. burgdorferi spirochetes in $3 \mathrm{ml}$ of culture media with different dilutions (1:5, 1:10, 1:25 and 1:50) of the compounds for $96 \mathrm{~h} \mathrm{[13].} \mathrm{The}$ samples were measured for fluorescence with a spectrophotometer (Perkin Elmer, 2030 Multilabel reader Victor" $\mathrm{x} 4$ ) every $24 \mathrm{~h}$.

RBs were induced as previously described [13]. Briefly, $15 \times 10^{6}$ B. burgdorferi spirochetes were incubated in sterile distilled $\mathrm{H}_{2} \mathrm{O}$ at $+37^{\circ} \mathrm{C}$ for $2 \mathrm{~h}$ before placing the cells into $3 \mathrm{ml}$ of fresh media and adding the same dilutions of each Biocidin compound as with the spirochete samples. Fluorescence measurements were done in the above-mentioned manner.

The MICs for both compounds with biofilms were determined by counting a $10 \mu \mathrm{l}$ area in a C-Chip DHC-N01 Disposable Haemocytometer (System Neubauer Improved, Digital Bio) at $0 \mathrm{~h}$ and $72 \mathrm{~h}$ post-treatment. Approximately 9000 biofilms in $3 \mathrm{ml}$ of fresh media were treated the previously mentioned dilutions of Biocidin compounds and incubated at $+37^{\circ} \mathrm{C}$ for $72 \mathrm{~h}$ after which the samples were counted. Cell clusters of over 10 cells were regarded as biofilms.

The minimum bacterial death (MBD) were determined as has been done previously with alterations [13]. Samples of $300 \mu$ from the MIC experiments from each pleomorphic form from the 72-h time point, respectively, were reseeded into $3 \mathrm{ml}$ of fresh media and incubated for 3 weeks at $+37^{\circ} \mathrm{C}$. At weeks 1 and 3 the change in $\mathrm{pH}$ (color change) of the media was used to indicate growth (yes/no) and microscopy (Leica CTR5500, Germany) was utilized at week 3 to confirm the observations.

\section{Time-kill experiments}

The rate of cell death of $B$. burgdorferi was analyzed by adding the MIC dilutions of the compounds (Liquid formula $=1: 10$; Liposomal formula $=1: 25$ ) to $6 \times 10^{6}$ spirochetes in $3 \mathrm{ml}$ of culture media and counting the cells using a C-Chip haemocytometer at 10, 20, 30, 60and 120 -min post-treatment. The means of three separate experiments were counted and Excel was used in producing the graph.

\section{Results}

\section{MIC and MBD experiments}

The MICs for Biocidin Liquid and Liposomal formulas with spirochetes, RBs and biofilms of B. burgdorferi were determined (Figure 1). The MICs for both compounds with each B. burgdorferi form in Figure 1 are highlighted with a black star. First, the MIC for the Liquid formula with spirochetes demonstrated 1:10 dilution (Figure 1A), while 1:25 dilution was enough with the Liposomal formula (Figure 1B), as these were the lowest dilutions at which no growth was observed. Second, the MICs for both formulas with RBs was determined to be the 1:50 dilution (Figure 1C and 1D), as the relative fluorescence values followed those of the negative controls. Last, the MICs of both formulas with biofilms were deduced by counting the biofilms in $10 \mu \mathrm{l}$ of each sample. Both compounds demonstrated an MIC of 1:10 dilution when cultured with biofilms (Figure 1E).

At $72 \mathrm{~h}$ time point of the MIC experiments, samples were put to fresh media and incubated for three weeks with the purpose of determining the MBDs for both compound with each $B$. burgdorferi pleomorphic form. Below table exemplifies the results from two separate experiments for each B. burgdorferi form (Table 1). Firstly, the Liquid formula demonstrated no growth with the 1:5 dilution during the whole threeweek time period in all of the bacterial forms. On the third week growth was observed in each pleomorphic form with the 1:10, 1:25 and 1:50 dilutions except the 1:10 dilution in RBs. Moreover, both spirochete and biofilm samples had growth with the 1:25 and 1:50 dilutions already at week one, while the RB samples showed none. Hence, the short-term dilutions of the Liquid formula for each pleomorphic form correlated those of the MIC results. However, only the 1:5 dilution resulted in no growth long-term with all pleomorphic forms.

Secondly, the results for the Liposomal formula followed those of the Liquid formula, although most of the spirochete samples have been examined only once and the third week of 1:10 dilution with RBs is completely missing because of inapplicable data. However, similarly to the Liquid formula, the Liposomal formula demonstrated no growth in the 1:5 dilution with each of the pleomorphic form. Furthermore, both spirochetes and biofilms indicated growth at week one already in the 1:25 and 1:50 dilutions, while RBs expressed no growth at week one in any of the dilutions. The first week MBD results for the Liposomal formula with RBs and biofilms corresponded to the MIC results. However, when cultured with spirochetes the 1:25 dilution already indicated growth at week one. Thus, contradicting the MIC for the Liposomal formula determined earlier.

\section{Time-kill experiments}

The effectiveness of the Biocidin compounds on B. burgdorferi spirochetes was determined in time-kill experiments (Figure 2). B. burgdorferi spirochetes were treated with the MICs of the compounds: 1:10 dilution for the Liquid formula; 1:25 for the Liposomal formula, and the cells were counted at 10,20,30,60- and 120-min posttreatment. Both compounds induced cell death of B. burgdorferi, with the Liquid formula being slightly more effective.

\section{Discussion}

Lyme borreliosis is currently the most common tick-borne disease in the Northern hemisphere (Stanek et al. 2012) [14]. Antibiotics are usually effective against Borrelia sp., however, persisting forms have been uncovered capable of resisting certain antibiotics $[6,15]$. Therefore, investigating novel possibilities for eliminating the persisting bacteria from the body is crucial. Here two dietary supplement compounds from Bio-Botanical Research Inc. were examined for their ability to eradicate the different pleomorphic forms of B. burgdorferi in vitro.

In recent years, the effects of several phytochemicals on Borrelia have been studied and found to be effective against the different pleomorphic forms of the bacteria [8-10,16-18]. For instance, Stevia whole leaf extract has been found to reduce the size and number of Borrelia biofilms [10]. Additionally, grapefruit seed extract has been demonstrated to work against Borrelia spirochetes and RBs [16]. Recently though, oregano oil was found to be highly active against persistent (7day growth) forms of Borrelia even at low concentrations [17]. Similarly, to previous studies, both of the compounds used here diminished the growth of all B. burgdorferi forms as was demonstrated by the MIC and MBD experiments. The active ingredients in both compounds vary from berry extracts to essential oils from plants. Interestingly, both of the compounds used here also have oregano oil, as well as, grapefruit seed extract as active agents in them.

Additionally, the number of spirochetes were drastically reduced after only $2 \mathrm{~h}$ post-treatment with the Biocidin compounds. This suggests that these compounds could offer a possible treatment for Lyme borreliosis, if not solely on their own but possibly when used 
$\mathbf{A}$

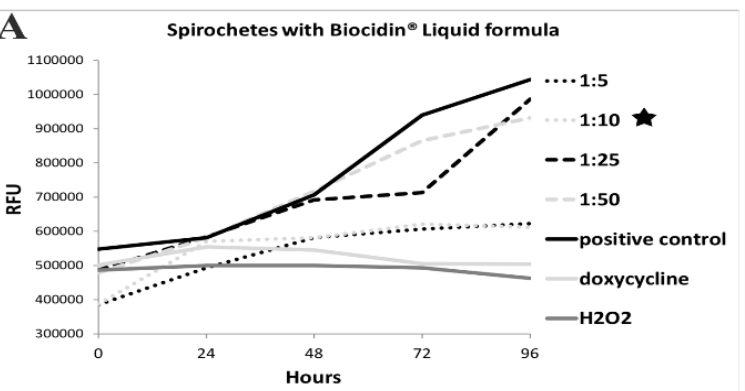

$\mathbf{C}$

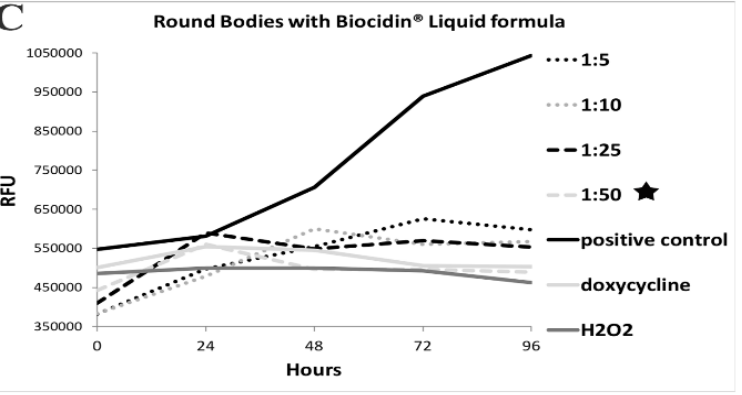

B

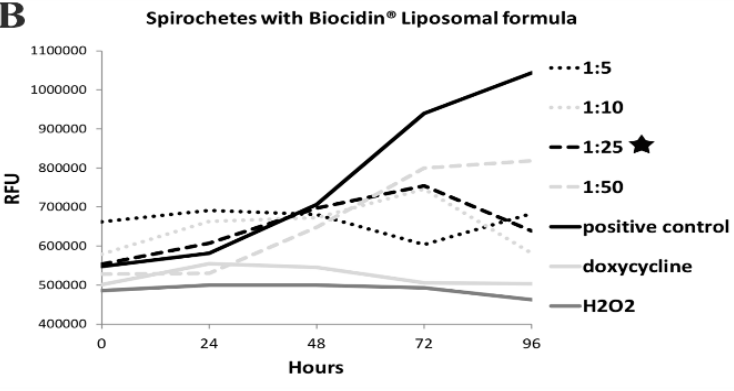

Dound Bodies with Biocidin ${ }^{\oplus}$ Liposomal formula

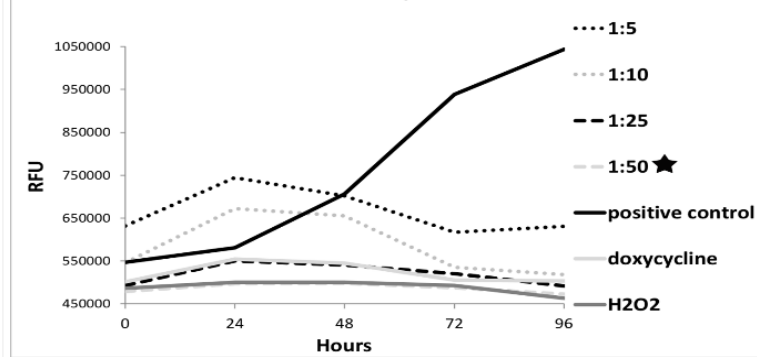

- Liquid formula

Q Liposomal formula

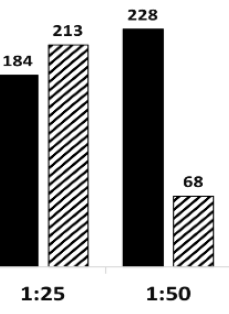

Figure 1. Minimum inhibitory concentrations of Biocidin ${ }^{\circledR}$ compounds with B. burgdorferi at various time points

$15 \times 10^{6}$ spirochetes (A and B) or Round Bodies (C and D) in $3 \mathrm{ml}$ of culture media were mixed with either Biocidin $\mathbb{B}$ Liquid (A and C) or Liposomal formula (B and D) in various dilutions $(1: 5,1: 10,1: 25$ and 1:50) for $96 \mathrm{~h}$. Panel E illustrates $9000 \mathrm{~B}$. burgdorferi biofilms in $3 \mathrm{ml}$ of culture media with the same dilutions as above of Liquid or Liposomal formula for $72 \mathrm{~h}$ Cells without a treatment were a positive control for growth, while $100 \mu \mathrm{g} / \mathrm{ml}$ of doxycycline and $0.02 \% \mathrm{H}_{2} \mathrm{O}_{2}$ were used as negative control for growth. Values for spirochetes and Round Bodies are means from two separate experiments. The values for biofilms with Liquid formula are from just one experiment, while those for the Liposomal formula are the means of three experiments. The black stars indicate the minimum inhibitory concentrations (MICs) for each compound for the different pleomorphic forms.

Table 1. Minimum bacterial death of $B$. burgdorferi spirochetes, Round Bodies and biofilms treated with Biocidin ${ }^{\circledR}$ compounds

Dilutions of 1:5, 1:10, 1:25 and 1:50 were used and growth was observed (yes/no) for three weeks. Cells without a treatment were a positive control for growth, while $100 \mu \mathrm{g} / \mathrm{ml}$ of doxycycline and $0.02 \% \mathrm{H}_{2} \mathrm{O}_{2}$ were used as negative control for growth. Results are mainly from two separate assays. Dashed lines indicate incomplete data, while the asterisk indicates only 1 repeat

\begin{tabular}{|c|c|c|c|c|c|c|}
\hline & \multicolumn{2}{|c|}{ Spirochetes } & \multicolumn{2}{|c|}{ Round Bodies } & \multicolumn{2}{|c|}{ Biofilms } \\
\hline & Week 1 & Week 3 & Week 1 & Week 3 & Week 1 & Week 3 \\
\hline Positive control & Yes & Yes & Yes & Yes & Yes & Yes \\
\hline Doxycycline & No & No & No & No & No & No \\
\hline $\mathrm{H}_{2} \mathrm{O}_{2}$ & No & No & No & No & No & No \\
\hline \multicolumn{7}{|l|}{ Liquid formula } \\
\hline $1: 5$ & No & No & No & No & No & No \\
\hline $1: 10$ & No & Yes & No & No & No & Yes \\
\hline $1: 25$ & Yes & Yes & No & Yes & Yes & Yes \\
\hline $1: 50$ & Yes & Yes & No & Yes & Yes & Yes \\
\hline \multicolumn{7}{|c|}{ Liposomal formula } \\
\hline $1: 5$ & No* & No* & No & No & No & No \\
\hline $1: 10$ & No & Yes* & No & --- & No & Yes \\
\hline $1: 25$ & Yes* & Yes* & No & Yes & Yes & Yes \\
\hline $1: 50$ & Yes* & Yes* & No & Yes & Yes & Yes \\
\hline
\end{tabular}

*only 1 repeat

--- data incomplete 


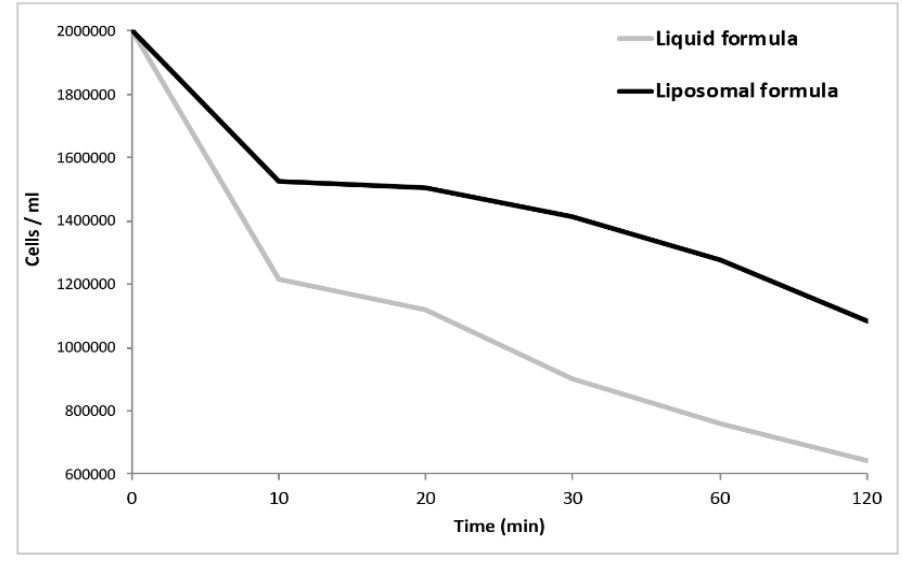

Figure 2. Rate of cell death after Biocidin ${ }^{\circledR}$ treatments

$6 \times 10^{6}$ Borrelia burgdorferi spirochetes in $3 \mathrm{ml}$ of media were treated with 1:10 Biocidin ${ }^{\circledR}$ Liquid formula or 1:25 Biocidin ${ }^{\circledR}$ Liposomal formula to check the rate of cell death. Cells were counted at 10, 20,30,60 and 120 min post-treatment. Represented are the mean values of three separate experiments. Both compounds drastically reduced B. burgdorferi spirochete count after $2 \mathrm{~h}$ post-treatment

synergistically with antibiotics as has been done before with other phytochemicals [19]. Naturally, studies involving antibiotics, as well as, RBs and biofilms should be conducted in order to realize the full potential of these compounds. Still, the promising results gained here for these two compounds indicate new possibilities to combat $B$. burgdorferi infections.

\section{Authorship and Contributorship}

All authors meet the criteria for authorship.

\section{Acknowledgements}

We'd like to acknowledge the Schwartz foundation for their continuous support of our research endeavors.

\section{Funding}

This work was supported by Bio-Botanical research Inc. However, the authors devised the study design, collected and analyzed the data, and prepared the manuscript without input from the company. The decision to publish was the authors.

\section{Conflicts of interest}

The authors have no conflicts of interest.

\section{References}

1. Burgdorfer W, Barbour AG, Hayes SF, Benach JL, Grunwaldt E (1982) Lyme disease-a tick-borne spirochetosis? Science 216: 1317-1319. [Crossref]

2. Meriläinen L, Herranen A, Schwarzbach A, Gilbert L (2015) Morphological and biochemical features of Borrelia burgdorferi pleomorphic forms. Microbiology 161: 516-527. [Crossref]

3. Murgia R, Cinco M (2004) Induction of cystic forms by different stress conditions in Borrelia burgdorferi. APMIS 112: 57-62. [Crossref]

4. Meriläinen L, Brander H, Herranen A, Schwarzbach A, Gilbert L (2016) Pleomorphic forms of Borrelia burgdorferi induce distinct immune responses. Microb.Infect [Crossref]

5. Stanek G, Wormser GP, Gray J, Strle F (2012) Lyme borreliosis. The Lancet 379: 461473 .

6. Sharma B, Brown AV, Matluck NE, Hu LT, Lewis K (2015) Borrelia burgdorferi, the Causative Agent of Lyme Disease, Forms Drug-Tolerant Persister Cells. Antimicrob. Agents Chemother 59: 4616-4624.
7. Sapi E, Kaur N, Anyanwu S, Luecke D, Datar A et al. (2011) Evaluation of in-vitro antibiotic susceptibility of different morphological forms of Borrelia burgdorferi. Infect Drug Resist 4: 97-113 [Crossref]

8. Feng J, Weitner M, Shi W, Zhang S, Sullivan D, et al. (2015) Identification of additional anti-persister activity against Borrelia burgdorferi from an FDA drug library. Antibiotics 4: 397-410. [Crossref]

9. Goc A, Niedzwiecki A, Rath M (2015) In vitro evaluation of antibacterial activity of phytochemicals and micronutrients against Borrelia burgdorferi and Borrelia garinii J.Appl.Microbiol 119: 1561-1572. [Crossref]

10. Theophilus PAS, Victoria MJ, Socarras K, Filush KR, Gupta K, et al. (2015) Effectiveness of Stevia rebaudiana whole leaf extract against the various morphologica forms of Borrelia burgdorferi in vitro. European Journal of Microbiology and Immunology 5: 268-280. [Crossref]

11. Moriarty TJ, Norman MU, Colarusso P, Bankhead T, Kubes P, et al. (2008) Real-time high resolution 3D imaging of the lyme disease spirochete adhering to and escaping from the vasculature of a living host. PLoS Pathog 4: e1000090. [Crossref]

12. Barbour AG (1984) Isolation and cultivation of Lyme disease spirochetes. Yale J.Biol. Med 57: 521-525. [Crossref]

13. Dever LL, Jorgensen JH, Barbour AG (1992) In vitro antimicrobial susceptibility testing of Borrelia burgdorferi: a microdilution MIC method and time-kill studies. $J$ Clin Microbiol 30: 2692-2697.

14. Hubalek Z (2009) Epidemiology of lyme borreliosis. Curr Probl Dermatol 37: 31-50. [Crossref]

15. Sapi E, Bastian SL, Mpoy CM, Scott S, Rattelle A, et al. (2012) Characterization of biofilm formation by Borrelia burgdorferi in vitro. PloS one 7: e48277. [Crossref]

16. Brorson $\varnothing$, Brorson S (2007) Grapefruit seed extract is a powerful in vitro agent against motile and cystic forms of Borrelia burgdorferi sensu lato. Infection 35: 206208. [Crossref]

17. Feng J, Zhang S, Shi W, Zubcevik N, Miklossy J, et al. (2017) Selective essential Oils from spice or culinary herbs have high activity against stationary Phase and Biofilm Borrelia burgdorferi. Frontiers in medicine 4: 169. [Crossref]

18. Goc A, Niedzwiecki A, Rath M (2017) Reciprocal cooperation of phytochemicals and micronutrients against typical and atypical forms of Borrelia spp. J.Appl.Microbiol. [Crossref]

19. Goc A, Niedzwiecki A, Rath M (2016) Cooperation of Doxycycline with Phytochemicals and Micronutrients Against Active and Persistent Forms of Borrelia sp. International Journal of Biological Sciences 12: 1093. [Crossref]

Copyright: (C)2018 Karvonen K. This is an open-access article distributed under the terms of the Creative Commons Attribution License, which permits unrestricted use, distribution, and reproduction in any medium, provided the original author and source are credited. 